\title{
On Demand Response Modeling and Optimization of Power in a Smart Grid
}

\author{
Olli Kilkki Kai Zenger \\ Department of Electrical Engineering and Automation, Aalto University School of Electrical Engineering, Finland \\ \{first\}. \{last\}@aalto.fi
}

\begin{abstract}
The electrical grid is under reform; the increasing volatile renewable energy production and distributed local generation compel the development of a future where the consumption of electricity can also participate in maintaining the production-consumption balance of the grid. There is a vast amount of recent research activity related to exploiting residential and industrial consumption elasticity. This paper presents a selected overview of various facets of modeling, optimizing and simulating the demand response potential and effects, especially with emergent soft computing methods. In addition, some illustrating examples are presented, where various relevant approaches from recent state-of-the-art research, including soft computing methods, are reviewed.
\end{abstract}

Keywords: Smart grid, optimization, soft computing

\section{Introduction}

The continuing increases in electricity consumption and volatile production in the form of renewable energy generation (Ela et al., 2011), are motivating the renewal of the electrical grid. This coming smart grid is envisioned to be more comprised of electronically controlled equipment than the current electromechanical foundations (Amin and Wollenberg, 2005). All the levels of the grid from the production, through transmission and distribution, to consumption will be fitted with metering and control devices to allow more granular and swift control (Farhangi, 2010).

One main part of the future smart grid is participation of electricity demand in maintaining grid health (Albadi and El-Saadany, 2007). The development of this demand response (DR) is motivated by the possible decrease in controllable production, as well as increase in local distributed generation (Castillo-Cagigal et al., 2011). In addition, local energy storages in residential or industrial facilities, such as electric vehicles (Kempton and Tomić, 2005), heat storages (Ericson, 2009) or batteries (Vytelingum et al., 2011), could be utilized for further enhancing the DR capabilities.

Before the actual distribution, electricity is bought and sold on various markets. These include, especially in Europe, some kind of day-ahead auction markets, where a price for the electricity is determined for all periods within the day (Imran and Kockar, 2014). Various intra-day mar- kets for further trading energy are also usually present. In addition, the real-time balance of the production and consumption of electricity is maintained by an assigned system operator (Kirschen and Strbac, 2004). The operator aims to ensure the balance by issuing imbalance fees to producers and consumers who do not adhere to their dayahead plans, purchasing regulating energy from various market participants and contracting reserves for continuous system balancing.

The demand-side could then be possibly utilized in various stages of market operation (Palensky and Dietrich, 2011). In the first place, smart appliances could enhance energy efficiency. With dynamic electricity prices, the time of use of appliances and other consumption could be shifted to minimize congestion during peak consumption. Then, with more dynamic control, the elasticity of the consumption could be utilized to participate e.g. in intra-day markets to shift consumption for agreed upon compensation. Finally, with more real-time communication and control capabilities, the demand-side could participate in various reserves.

These different options for market participation, in conjunction with the more distributed nature of electricity production and grid health maintenance, provide an ample range of problems and possibilities. The relevant control problems required for demand response participation have to be modeled carefully, due to their dual nature involving the competing objectives related to grid stability and level of comfort (Callaway and Hiskens, 2011). In addition, the amount of uncertainties is going to even increase (Varaiya et al., 2011), and thus has to be taken into account both in the modeling and optimization stages, as well as the final simulations. Various traditional and more novel approaches can be found to offer solutions to the aforementioned problems. Especially soft computing and computational intelligence methods are seen to be able to respond to the various complicated problems at hand (Venayagamoorthy, 2011).

Similarly to previous reviews on the subject of computational intelligence methods in the smart grid context (Venayagamoorthy, 2011), this paper presents the possibilities of soft computing methods, but also with consideration for traditional approaches. This paper presents a selected overview of modeling, optimizing and simulating demand response potential, in addition to some exam- 
ples. In the examples, various relevant approaches from recent state-of-the-art research, including soft computing methods, are reviewed, and discussion presented on possible future research directions. At first, we outline the elements and actors to be modeled related to demand-side management. Then we delve into the control of those elements with planning consumptions schedules and then more real-time control related to the grid frequency, while showing some examples. Finally, some discussion on the findings, and challenges and possibilities of the demand response are presented.

\section{Modeling demand-side manage- ment}

There are multiple facets to modeling the effects and potential of demand response in a smart grid. Traditionally electricity delivery and consumption has been a hierarchical system, with electricity provided by large power plants and consumed by customers behind the transmission and distribution networks (Kirschen and Strbac, 2004). However, with the coming smarter electricity grid, the direction of electricity transfer can vary and role of the various actors is expanded (Amin and Wollenberg, 2005). The objectives of these actors and their communication have to be properly modeled, especially if they are to operate in an independent manner. The main players in the electricity market to be modeled include the electricity markets, electricity producers, independent system operator, retailers and finally electricity consumers (Kirschen and Strbac, 2004). In addition, the actual grid, or parts of it, might have to be modeled depending on the effects that are under study.

\subsection{Markets}

Auction-based electricity markets facilitate the trading of electricity by aggregating production and consumption bids of electricity, and determining an hourly price (Kirschen and Strbac, 2004). Electricity retailers participate on these markets on behalf of the end-consumers by aggregating their consumption. If the customer electricity consumption schedules can be affected, the aggregating retailers can reduce electricity acquisition costs by acquiring more electricity during lower priced hours.

The markets can be modeled by the retailers by assuming that their effect on the market is minimal, and modeling the resulting electricity price as a stochastic process (Zugno et al., 2013). The model for the price can take into account various influences, including climate and weather data, hydro-power availability and electricity demand (Vehviläinen and Pyykkönen, 2005). In addition, the uncertainties in the realizations manifest as normal variation as well as larger price peaks (Voronin et al., 2014). Various methods can be utilized in modeling and forecasting the prices, such as ARMA, GARCH, neural networks and GMMs (Voronin et al., 2014). Alternatively, the price of electricity can be assumed to directly reflect the amount of demand. For example, in many studies the cost of electricity is set to rise quadratically w.r.t. the amount of total consumption (Mohsenian-Rad et al., 2010; Samadi et al., 2010).

\subsection{Actors}

The aggregating retailer in the context of a smart grid is often referred to as a virtual power plant (VPP), which can aggregate the consumption as well as elasticity of the consumption, and possible distributed production, of multiple industrial or residential customers (Pudjianto et al., 2007). The VPP has to be modeled with both the technical and commercial roles in mind (Pudjianto et al., 2007). When participating in the various markets and providing system balancing services, the VPP has to be able to affect the consumption profiles of its managed customers. There are various alternative approaches for controlling the consumption, which are discussed in more detail in the following Section 3.

Conversely, the consumers are mainly concerned with maintaining their level-of-comfort or production deadlines. In addition, depending on the contract made with the VPP, they have to be able to shift their consumption either under command or voluntarily through compensation (Albadi and El-Saadany, 2007). The customers can have various loads that they use their electricity on. For example, various thermostatically controlled loads such as direct heating or refrigerators could be modeled and utilized for various grid maintenance activities (Callaway and Hiskens, 2011). One general application involves a stochastic load and some intermediate energy storage, such as storage space heating (Ali et al., 2014). Ali et al. (Ali and Koivisto, 2013) devised such a model for a particular consumer $n$, where the storage charging $P_{n, t}$ is constrained by the heating demand $Q_{n, t}$ and storage limits $C_{\max , n}$ :

$$
\begin{gathered}
0 \leq P_{n, t} \leq P_{\max , n} \\
\sum_{k=1}^{t}\left(P_{n, k}-Q_{n, k}\right) \Delta t \geq-C_{n, 0} \\
\sum_{k=1}^{t}\left(P_{n, k}-Q_{n, k}\right) \Delta t \leq C_{\max , n}-C_{n, 0}
\end{gathered}
$$

A more detailed model would be required for industrial applications for providing demand response (Ding et al., 2014), Examples of models for industrial applications include production planning with detailed constraints for operating mode switching with proper ramping behaviour by Mitra et al. (Mitra et al., 2012), and the work by Castro et al. which takes into account discrete events such as due dates and utility availabilities (Castro et al., 2009).

\subsection{Other elements}

In addition, other elements have to be taken into account when modeling demand response potential and effects on a larger scale. Locational aspects affect the realization of demand response on a larger scale. Among 
these locational aspects are the communication networks enabling coordination of the demand response (Güngör et al., 2011). The communication infrastructure of the smart grid is envisioned to consist of a combination of existing networks and technologies (Güngör et al., 2011), where the latencies and other characteristics are important considerations ( $\mathrm{Lu}$ et al., 2013). These communication channels can be included in the models by integrating external communication network simulators (Mets et al., 2011), or more directly by utilizing various probability distributions for the relevant parameters (Kilkki et al., 2014). In addition, constraints on locational grid limits might have to be taken into account (Wang et al., 2015).

\section{Controlling consumption}

Responsive demand can be utilized for various system balancing activities on various timescales (Palensky and Dietrich, 2011). Related to the various services that the VPP can offer to the grid and markets, are the ways in which the control can be exerted on the consumer loads. The main classification of different demand response programs, based on the ways the consumers are incentivized and contracted to react, can be started with a split into incentive based programs and price based programs (Albadi and El-Saadany, 2007). In incentive based programs the incentives can be paid out either by directly assuming them into the consumer contracts or by determining them with on market based methods, e.g. bidding.

In contrast, with price-based programs, the VPP or other controlling entity would choose a dynamic price for the electricity it is offering to its customers (Albadi and El-Saadany, 2007). The type of dynamic price can vary from day-ahead chosen time-of-use prices, to critical peak pricing, or real-time pricing.

\subsection{Optimizing scheduling}

Two major areas of research involve planning of consumption and charging schedules, and then dispatching the actual consumption. The main objectives usually include taking into account the utility of the consumers, cost of electricity and any possible grid constraints. Traditional optimization methods such as linear programming (LP), mixed-integer linear programming (MILP) or quadratic programming $(\mathrm{QP})$, or a mixture thereof, are routinely utilized in obtaining consumption schedules. Electric vehicle charging aggregation is a thoroughly researched topic, where the charging schedules can be optimized for example w.r.t. uncertain renewable generation (Pantoš, 2011), or taking into account the risks involved in the costs and profits (Momber et al., 2015).

In addition, heating is another large part of electricity consumption which could be temporarily deferred. For example, Nguyen et al. (Nguyen and Le, 2014) propose a method for planning heating schedules w.r.t the dayahead electricity acquisition costs, while considering various uncertainties. In Section 2.2 we detailed a simplified model for an energy storage in conjunction with a heating load, and we have previously also proposed (Kilkki et al., 2015a) an optimization algorithm for its charging schedules. We utilized a genetic algorithm to optimize the dynamic price $K_{t}$ an aggregator charges from its independent consumers, in order to achieve a desirable aggregate consumption profile. The consumers aim to minimize their electricity costs by optimizing

$$
\min _{\text {w.r.t. } P} \sum_{t=1}^{H} K_{t} P_{n, t}
$$

while holding the constraints (1)-(3). The aggregators profits comprise of the expectation function

$$
\max \mathbb{E}\left[\sum_{t=1}^{H} K_{t}\left(P_{t}+L_{t}\right)-K_{t}^{s} E_{t}-\pi_{t}^{\uparrow} \Delta E_{t}^{\uparrow}+\pi_{t}^{\downarrow} \Delta E_{t}^{\downarrow}\right]
$$

where $P_{t}$ is the aggregate consumption, $K_{t}^{s}$ the day-ahead market price, $\pi_{t}^{\uparrow / \downarrow}$ and $\Delta E_{t}^{\uparrow / \downarrow}$ the imbalances, costs and payments for positive and negative deviations from the planned schedules, respectively. The optimization aims to minimize these costs as well as the acquisition costs, while maximizing profit that can be acquired from the consumers. The imbalances that are however realized during the day, were proposed to be minimized by offering favorable changes to the electricity price of the consumers for them to shift their consumption. The price discount amount and percentage of consumers to offer to were also optimized using genetic algorithms with the to-bemaximized function defined as

$$
\max _{\text {w.r.t. } \Delta K, n} \pi_{t}^{\uparrow / \downarrow}\left(\Delta E_{t}^{\uparrow / \downarrow}+P_{t}^{d}-P_{t}\right)+\sum_{k=t}^{H} n\left(K_{k}^{d} P_{k}^{d}-K_{k} P_{k}\right)
$$

where $K_{k}^{d}$ is the discounted price (with discounts $\Delta K \leq 0$ ) and $P_{k}^{d}$ the consumption of the consumers after the discount. Figure 1 shows results obtained from the aforementioned optimizations, when discounts were given at hours 3, 14 and 20. The upper subfigure shows the realized load consisting of the storage space heater charging and other inflexible consumption sources. The lower subfigure them shows the distribution of realized imbalances from the acquired electricity schedule, that occur due to various uncertainties. It can be seen how during the hours with discounts, the amount of imbalances is reduced significantly, resulting in positive effects for the aggregator, consumers, and the grid.

An evolutionary approach has also been utilized for various other problems, such as by Logenthiran et al. for scheduling general shiftable loads (Logenthiran et al., 2012). In addition to genetic algorithms, various other soft computing methods have been utilized in planning consumption schedules (Venayagamoorthy, 2011). For example, Soares et al. developed a day-ahead scheduling method for electric vehicles, with considerations for demand response. The resulting mixed-integer nonlinear programming problem was solved utilizing traditional 


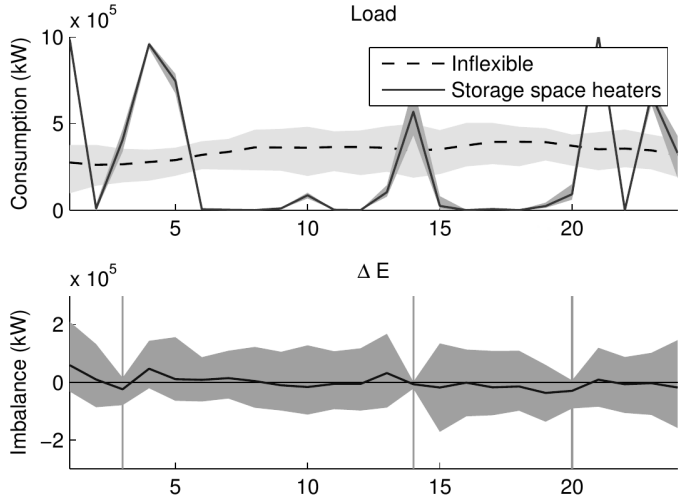

Figure 1. Realized charging and inflexible consumption as well as remaining imbalances from the acquired schedules after discounts. (Kilkki et al., 2015a)

nonlinear optimization methods but was found to be significantly faster to solve with a particle swarm optimization approach (Soares et al., 2013). Various fuzzy logic (Dubey et al., 2015; Ma and Mohammed, 2014) and neural network solutions (Siano et al., 2012), and combinations thereof (Ozturk et al., 2013; Shahgoshtasbi and Jamshid, 2011) have been also employed, in optimizing day-ahead electricity consumption schedules.

\subsection{Controlling (via) frequency}

The instantaneous balance of total aggregate production and consumption in the electrical grid can be inferred to some extent from the frequency of the grid (Kundur et al., 2004). The system operator is in charge of maintaining this frequency close to its nominal value. The maintenance is performed by purchasing regulating power to minimize larger imbalances, and contracting either power plants or consumption for providing continuous reserves. Involving the consumption in this frequency control is widely researched with applications to power plants such as hydro (Doolla and Bhatti, 2006) and wind turbines (Ramtharan et al., 2007), as well as to demand.

In its most simplest form, frequency regulation can be implemented by implementing the traditional droop control with consumers increasing and decreasing their loads w.r.t. to the deviation of grid frequency from its nominal value (Palensky and Dietrich, 2011). Figure 2 displays the traditional droop of a frequency controller, where $P_{r}$ is the amount of reserves promised, $\Delta f_{d b}$ is the deadband of the frequency deviations and $\Delta f_{\max }$ the frequency deviation where the maximal control has to be exerted. Thermostatical loads can be similarly co-ordinated to perform the droop control by issuing frequency limits after which they turn their loads on or off, respectively.

Molina-García et al. (Molina-Garcia et al., 2011) proposed a similar method while including time characteristics to the frequency control thresholds. In their proposed approach, different load types were given different characteristics on how fast to respond to varying sizes of deviations of varying lengths. For an alternative approach, Call-

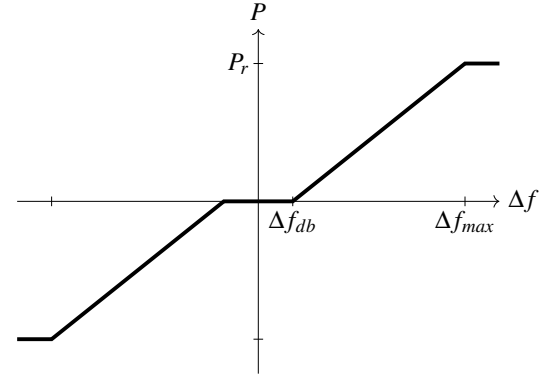

Figure 2. Droop.

away proposed an aggregated continuous control method for a group of thermostatically controlled loads (Callaway, 2009), which could be utilized for frequency regulation. The control was achieved by directly altering the temperature setpoints of the thermostats, while maintaining an aggregated load model of all of the loads under control. Callaway and Hiskens also proposed similar methods for energy storage charging devices, such as electric vehicles, where a hysteresis-based on-off charging cycle is manipulated by adjusting the deadbands of the cycles (Callaway and Hiskens, 2011).

In addition to the more traditional approaches, the various proposed methods have utilized soft computing solutions. A fuzzy logic based frequency control method was developed by Datta (Datta, 2014), where electric vehicle charging was controlled to provide frequency regulation. Similar fuzzy methods were also utilized for photovoltaics inverter control (Datta and Senjyu, 2013). Bevrani \& Shokoohi utilized a fuzzy approach as well in their work (Bevrani and Shokoohi, 2013), where they developed a model-free generalized droop control scheme for microgrids. The model-free controller was obtained by a neural network approach, where the generated network was trained with historical training data to achieve the desired co-ordinated droop control. Debbarma and Dutta propose (Debbarma and Dutta, 2016) a fractional order controller for controlling the charging of electric vehicles. A flower pollination algorithm was then utilized for tuning the actual parameters of the controller. The flower pollination algorithm is a metaheuristic optimization algorithm (Yang, 2012), which takes inspiration from the pollination process of flowers with elements such as seperation to local and global pollination and a random walk based on the Lévy flight.

There have also been multiple studies on taking into account frequency regulation capacity in day-ahead planning and market operation. For example, the author has previously proposed an approach for scheduling the charging of energy storages utilized in heating, while including participation in frequency reserve markets (Kilkki et al., 2015b). Conversely, Yuen et al. proposed an algorithm for provisioning reserves from multiple microgrids (Yuen et al., 2011). Vayá and Andersson developed a method for planning the day-ahead charging schedules of an aggrega- 
tor, while simultaneously optimizing for frequency regulation participation (Vayá and Andersson, 2013), as well as a real-time dispatch algorithm for the regulation. The aggregated vehicle charging patterns utilized in the optimization of the day-ahead schedules were obtained by utilizing a co-evolutionary algorithm for optimization. Similarly optimizing electric vehicle charging was also proposed by Sortomme and El-Sharkawi (Sortomme and ElSharkawi, 2012). Their algorithm also takes into account reserve participation, and additionally includes in the optimization the uncertainties in departure times of the vehicles.

\section{Discussion and conclusions}

The modernification of the electrical grid brings with it many challenges. As the grid transforms from a more hierarchical system to a more diverse collection of more dynamic actors, the control and its various effects have to be carefully considered. However, many opportunities also arise as the penetration of relevant ICT devices can enable responsive demand. Both residential and industrial demand could be included in various demand-side management programs, ranging from day-ahead scheduling and market bidding, to more real-time applications such as participation in frequency reserves. For modeling and optimizing the responsiveness of the demand, various methods can be utilized. Traditional methods can provide a more predictable response, but with the increase in the complexity of the system, various soft computing methods become attractive alternatives. We found multiple proposed applications of genetic algorithms and fuzzy methods in scheduling the consumption, as well as in coordinating frequency control. Soft computing methods we found to be utilizable in the optimization of scheduling, as well as tuning of more real-time control. In addition, with the various uncertainties involved in the optimization and control of consumption, various methods are required for identifying the time-series models related to the uncertainties. Computational intelligence methods are again often utilized.

\section{References}

MH Albadi and EF El-Saadany. Demand response in electricity markets: An overview. In IEEE Power Engineering Society General Meeting, volume 2007, pages 1-5, 2007.

Mubbashir Ali and Matti Koivisto. Optimizing the DR control of electric storage space heating using LP approach. International Review on Modelling and Simulations (IREMOS), 6(3): 853-860, 2013.

Mubbashir Ali, Juha Jokisalo, Kai Siren, and Matti Lehtonen. Combining the demand response of direct electric space heating and partial thermal storage using LP optimization. Electric Power Systems Research, 106:160-167, 2014.

S Massoud Amin and Bruce F Wollenberg. Toward a smart grid: power delivery for the 21 st century. Power and energy Magazine, IEEE, 3(5):34-41, 2005.
Hassan Bevrani and Shoresh Shokoohi. An intelligent droop control for simultaneous voltage and frequency regulation in islanded microgrids. Smart Grid, IEEE Transactions on, 4 (3):1505-1513, 2013.

Duncan S Callaway. Tapping the energy storage potential in electric loads to deliver load following and regulation, with application to wind energy. Energy Conversion and Management, 50(5):1389-1400, 2009.

Duncan S Callaway and Ian A Hiskens. Achieving controllability of electric loads. Proceedings of the IEEE, 99(1):184-199, 2011.

Manuel Castillo-Cagigal, Estefanía Caamaño-Martín, Eduardo Matallanas, Daniel Masa-Bote, A Gutiérrez, F MonasterioHuelin, and J Jiménez-Leube. PV self-consumption optimization with storage and active DSM for the residential sector. Solar Energy, 85(9):2338-2348, 2011.

Pedro M Castro, Iiro Harjunkoski, and Ignacio E Grossmann. New continuous-time scheduling formulation for continuous plants under variable electricity cost. Industrial \& engineering chemistry research, 48(14):6701-6714, 2009.

Manoj Datta. Fuzzy logic based frequency control by V2G aggregators. In Power Electronics for Distributed Generation Systems (PEDG), 2014 IEEE 5th International Symposium on, pages 1-8. IEEE, 2014.

Manoj Datta and Tomonobu Senjyu. Fuzzy control of distributed PV inverters/energy storage systems/electric vehicles for frequency regulation in a large power system. Smart Grid, IEEE Transactions on, 4(1):479-488, 2013.

Sanjoy Debbarma and Arunima Dutta. Utilizing electric vehicles for LFC in restructured power systems using fractional order controller. Smart Grid, IEEE Transactions on, 2016.

Yue Min Ding, Seung Ho Hong, and Xiao Hui Li. A demand response energy management scheme for industrial facilities in smart grid. Industrial Informatics, IEEE Transactions on, 10(4):2257-2269, 2014.

Suryanarayana Doolla and TS Bhatti. Load frequency control of an isolated small-hydro power plant with reduced dump load. Power Systems, IEEE Transactions on, 21(4):19121919, 2006.

Hari Mohan Dubey, Manjaree Pandit, and BK Panigrahi. Hybrid flower pollination algorithm with time-varying fuzzy selection mechanism for wind integrated multi-objective dynamic economic dispatch. Renewable Energy, 83:188-202, 2015.

Erik Ela, Michael Milligan, and Brendan Kirby. Operating reserves and variable generation. Contract, 303:275-3000, 2011.

Torgeir Ericson. Direct load control of residential water heaters. Energy Policy, 37(9):3502-3512, 2009.

Hassan Farhangi. The path of the smart grid. Power and energy magazine, IEEE, 8(1):18-28, 2010. 
Vehbi C Güngör, Dilan Sahin, Taskin Kocak, Salih Ergüt, Concettina Buccella, Carlo Cecati, and Gerhard P Hancke. Smart grid technologies: communication technologies and standards. Industrial informatics, IEEE transactions on, 7(4): 529-539, 2011.

Kashif Imran and Ivana Kockar. A technical comparison of wholesale electricity markets in North America and Europe. Electric Power Systems Research, 108:59-67, 2014.

Willett Kempton and Jasna Tomić. Vehicle-to-grid power implementation: From stabilizing the grid to supporting largescale renewable energy. Journal of Power Sources, 144(1): 280-294, 2005.

Olli Kilkki, A Kangasrääsiö, Raimo Nikkilä, Antti Alahäivälä, and Ilkka Seilonen. Agent-based modeling and simulation of a smart grid: A case study of communication effects on frequency control. Engineering Applications of Artificial Intelligence, 33:91-98, 2014.

Olli Kilkki, Antti Alahaivala, and Ilkka Seilonen. Optimized control of price-based demand response with electric storage space heating. Industrial Informatics, IEEE Transactions on, 11(1):281-288, 2015a.

Olli Kilkki, Christian Giovanelli, Ilkka Seilonen, and Valeriy Vyatkin. Optimization of decentralized energy storage flexibility for frequency reserves. In Industrial Electronics Society, IECON 2015-41st Annual Conference of the IEEE, pages 002219-002224. IEEE, 2015b.

Daniel Kirschen and Goran Strbac. Fundamentals of Power System Economics. Wiley Online Library, 2004.

Prabha Kundur, John Paserba, Venkat Ajjarapu, Göran Andersson, Anjan Bose, Claudio Canizares, Nikos Hatziargyriou, David Hill, Alex Stankovic, Carson Taylor, et al. Definition and classification of power system stability ieee/cigre joint task force on stability terms and definitions. Power Systems, IEEE Transactions on, 19(3):1387-1401, 2004.

Thillainathan Logenthiran, Dipti Srinivasan, and Tan Zong Shun. Demand side management in smart grid using heuristic optimization. Smart Grid, IEEE Transactions on, 3(3): 1244-1252, 2012.

Xiang Lu, Wenye Wang, and Jianfeng Ma. An empirical study of communication infrastructures towards the smart grid: Design, implementation, and evaluation. Smart Grid, IEEE Transactions on, 4(1):170-183, 2013.

Tan Ma and Osama A Mohammed. Optimal charging of plug-in electric vehicles for a car-park infrastructure. Industry Applications, IEEE Transactions on, 50(4):2323-2330, 2014.

Kevin Mets, Tom Verschueren, Chris Develder, Tine L Vandoorn, and Lieven Vandevelde. Integrated simulation of power and communication networks for smart grid applications. In Computer Aided Modeling and Design of Coтmunication Links and Networks (CAMAD), 2011 IEEE 16th International Workshop on, pages 61-65. IEEE, 2011.

Sumit Mitra, Ignacio E Grossmann, Jose M Pinto, and Nikhil Arora. Optimal production planning under time-sensitive electricity prices for continuous power-intensive processes. Computers \& Chemical Engineering, 38:171-184, 2012.
Amir-Hamed Mohsenian-Rad, Vincent WS Wong, Juri Jatskevich, Robert Schober, and Alberto Leon-Garcia. Autonomous demand-side management based on game-theoretic energy consumption scheduling for the future smart grid. Smart Grid, IEEE Transactions on, 1(3):320-331, 2010.

Angel Molina-Garcia, François Bouffard, and Daniel S Kirschen. Decentralized demand-side contribution to primary frequency control. Power Systems, IEEE Transactions on, 26 (1):411-419, 2011.

Ilan Momber, Afzal Siddiqui, Tomas Gomez San Roman, and Lennart Soder. Risk averse scheduling by a pev aggregator under uncertainty. Power Systems, IEEE Transactions on, 30 (2):882-891, 2015.

Duong Tung Nguyen and Long Bao Le. Optimal bidding strategy for microgrids considering renewable energy and building thermal dynamics. Smart Grid, IEEE Transactions on, 5 (4):1608-1620, 2014.

Yusuf Ozturk, Datchanamoorthy Senthilkumar, Sudhakar Kumar, and Gene Lee. An intelligent home energy management system to improve demand response. Smart Grid, IEEE Transactions on, 4(2):694-701, 2013.

Peter Palensky and Dietmar Dietrich. Demand side management: Demand response, intelligent energy systems, and smart loads. Industrial Informatics, IEEE Transactions on, 7(3):381-388, 2011.

Miloš Pantoš. Stochastic optimal charging of electric-drive vehicles with renewable energy. Energy, 36(11):6567-6576, 2011.

Danny Pudjianto, Charlotte Ramsay, and Goran Strbac. Virtual power plant and system integration of distributed energy resources. Renewable power generation, IET, 1(1):10-16, 2007.

G Ramtharan, Janaka Bandara Ekanayake, and Nicholas Jenkins. Frequency support from doubly fed induction generator wind turbines. Renewable Power Generation, IET, 1(1):3-9, 2007.

Pedram Samadi, Amir-Hamed Mohsenian-Rad, Robert Schober, Vincent WS Wong, and Juri Jatskevich. Optimal real-time pricing algorithm based on utility maximization for smart grid. In Smart Grid Communications (SmartGridComm), 2010 First IEEE International Conference on, pages 415420. IEEE, 2010.

Dariush Shahgoshtasbi and Mo Jamshid. Energy efficiency in a smart house with an intelligent neuro-fuzzy lookup table. In System of Systems Engineering (SoSE), 2011 6th International Conference on, pages 288-292. IEEE, 2011.

Pierluigi Siano, Carlo Cecati, Hao Yu, and Janusz Kolbusz. Real time operation of smart grids via FCN networks and optimal power flow. Industrial Informatics, IEEE Transactions on, 8 (4):944-952, 2012.

Joao Soares, Hugo Morais, Tiago Sousa, Zita Vale, and Pedro Faria. Day-ahead resource scheduling including demand response for electric vehicles. Smart Grid, IEEE Transactions on, 4(1):596-605, 2013. 
Eric Sortomme and Mohamed A El-Sharkawi. Optimal scheduling of vehicle-to-grid energy and ancillary services. Smart Grid, IEEE Transactions on, 3(1):351-359, 2012.

Pravin P Varaiya, Felix F Wu, and Janusz W Bialek. Smart operation of smart grid: Risk-limiting dispatch. Proceedings of the IEEE, 99(1):40-57, 2011.

M González Vayá and Göran Andersson. Combined smartcharging and frequency regulation for fleets of plug-in electric vehicles. In IEEE Power and Energy Society General Meeting, volume 2013, 2013.

Iivo Vehviläinen and Tuomas Pyykkönen. Stochastic factor model for electricity spot price - the case of the nordic market. Energy Economics, 27(2):351-367, 2005.

Ganesh Kumar Venayagamoorthy. Dynamic, stochastic, computational, and scalable technologies for smart grids. Computational Intelligence Magazine, IEEE, 6(3):22-35, 2011.

Sergey Voronin, Jarmo Partanen, and Tuomo Kauranne. A hybrid electricity price forecasting model for the nordic electricity spot market. International Transactions on Electrical Energy Systems, 24(5):736-760, 2014.

Perukrishnen Vytelingum, Thomas D Voice, Sarvapali D Ramchurn, Alex Rogers, and Nicholas R Jennings. Theoretical and practical foundations of large-scale agent-based microstorage in the smart grid. Journal of Artificial Intelligence Research, 42(1):765-813, 2011.

Kun Wang, Zhiyou Ouyang, Rahul Krishnan, Lei Shu, and Lei He. A game theory-based energy management system using price elasticity for smart grids. Industrial Informatics, IEEE Transactions on, 11(6):1607-1616, 2015.

Xin-She Yang. Flower pollination algorithm for global optimization. In Unconventional computation and natural computation, pages 240-249. Springer, 2012.

Cherry Yuen, Alexandre Oudalov, and Adrian Timbus. The provision of frequency control reserves from multiple microgrids. Industrial Electronics, IEEE Transactions on, 58(1): 173-183, 2011.

Marco Zugno, Juan Miguel Morales, Pierre Pinson, and Henrik Madsen. A bilevel model for electricity retailers' participation in a demand response market environment. Energy Economics, 36:182-197, 2013. 